\title{
Modelado de índices de competencia para Araucaria angustifolia en dos sitios en el sur de Brasil
}

\author{
Modeling competition indices for Araucaria angustifolia \\ at two sites in southern Brazil
}

\author{
Emanuel Arnoni Costa ${ }^{\text {a*, }}$, César Augusto Guimarães Finger ${ }^{\text {b,c }}$, Paulo Renato Schneider ${ }^{\text {b }}$, \\ André Felipe Hess ${ }^{d}$, Veraldo Liesenberg ${ }^{d}$, Cristine Tagliapietra Schons ${ }^{\mathrm{e}}$ \\ *Autor de correspondencia: ${ }^{a}$ Universidade Federal de Uberlândia, Instituto de Ciências Agrárias, Monte Carmelo, \\ Minas Gerais, Brasil, tel.: +55 34 99232-3787, emanuelarnonicost@gmail.com \\ ${ }^{\mathrm{b}}$ Universidade Federal de Santa Maria, Departamento de Ciências Florestais, Av. Roraima, 1000 Santa Maria, \\ Rio Grande do Sul, Brasil. \\ c Universidade Tecnológica Federal do Paraná, Estrada para Boa Esperança Km 4, Dois Vizinhos, Paraná, Brasil. \\ ${ }^{d}$ Universidade do Estado de Santa Catarina, Centro de Ciências Agroveterinárias, Av. Luiz de Camoes, \\ Lages, Santa Catarina, Brasil. \\ e Universidade Federal de Santa Maria, Programa de Pós Graduação em Engenharia Florestal, Av. Roraima, 1000, \\ Santa Maria, Rio Grande do Sul, Brasil.
}

\begin{abstract}
SUMMARY
This study aimed at modeling competition indices for individual trees of Araucaria angustifolia in natural forests. A total of 397 objective trees were sampled in Lages and São Francisco de Paula municipalities. Through a non-linear regression analysis, 22 distance-independent and distance-dependent competition indices were modeled based on the diameter at breast height (d) and relative height (hrel: ratio between the total height of the objective tree and the maximum potential height estimated for the same diameter). The adjusted equations of the indices according to $\mathrm{d}$ of the objective tree achieved values of $\mathrm{R}^{2} \leq 0.546(\mathrm{Syx} \% \geq 15.0)$. The independent variable hrel improved the estimates, explaining up to $63 \%$ of the variation in the data (Syx\% $\geq 14.6)$. In general, competition indices - which consider in the calculation only the variables $\mathrm{d}$ of the objective tree, $\mathrm{d}$ of the competitors, its horizontal distance and eventually the height variable- showed superior performance. Therefore, they are indicated for use in growth modeling of A. angustifolia, due to superior facility of application in natural forests. The developed equations showed to be good representations of the degree of competition at the level of the individual tree of A. angustifolia for the two evaluated sites, measuring only the diameter or including the height of the objective tree for more accurate precision. The expansion of research to other sites along the distribution of the species will confirm the application of these equations to their full extent. In addition, this work contributes to the inclusion of competition indices in modeling of individual trees in uneven-aged mixed forests.
\end{abstract}

Key words: Parana pine; biometric modeling; competition among trees.

\section{RESUMEN}

El presente estudio modeló índices de competencia para árboles individuales de Araucaria angustifolia en bosque nativo. Fueron muestreados 397 árboles objetivo en los municipios de Lages y São Francisco de Paula. Mediante análisis de regresión no linear fueron modelados 22 índices de competencia independientes y dependientes de la distancia en función del diámetro a la altura del pecho y altura relativa (razón entre la altura total del árbol objetivo y la máxima altura potencial estimada para el mismo diámetro). Las ecuaciones ajustadas de los índices en función del diámetro del árbol objetivo alcanzaron valores de $\mathrm{R}^{2} \leq 0,546(\mathrm{Syx} \% \geq 15,0)$. La altura relativa mejoró las estimaciones, explicando hasta el $63 \%$ de la variación de datos (Syx\% $\geq 14,6)$. En general, los índices de competencia que consideraron solo los diámetros del árbol objetivo y de los competidores, sus distancias horizontales, y eventualmente la altura, presentaron desempeño superior, siendo así indicados para el modelado del crecimiento de A. Angustifolia, debido a la mayor facilidad de aplicación en bosque nativo. Las ecuaciones desarrolladas representaron bien el grado de competencia a nivel del árbol individual de $A$. angustifolia para los dos sitios evaluados, midiendo solo el diámetro, o incluyendo también la altura del árbol objetivo para una mayor precisión. La expansión de la investigación a otros sitios a lo largo de la distribución de las especies permitirá confirmar la aplicación de estas ecuaciones en toda su amplitud. Además, este trabajo contribuye a la inclusión de índices de competencia en el modelado de árboles individuales en bosques mixtos disetáneos.

Palabras clave: pinheiro-do-Paraná, modelado biométrico, competencia entre árboles. 


\section{INTRODUCCIÓN}

La competencia de los árboles es una interacción fundamental dentro de la comunidad de plantas y un proceso muy complejo que involucra muchos mecanismos (Kuehne et al. 2019). Ocurre cuando dos o más árboles individuales intentan capturar los mismos recursos (como la luz solar, el agua y los nutrientes) mientras los suministros de recursos están por debajo de los niveles capaces de sostener un crecimiento óptimo de ellos (Craine y Dybzinski 2013, Zhang et al. 2019). Esto siempre conduce a una reducción en la supervivencia, el crecimiento y/o la reproducción (Burkhart y Tomé 2012).

En este sentido, la competencia es llamada intraespecífica cuando ocurre entre seres de la misma especie, e interespecífica cuando ocurre entre especies distintas (Weiskittel et al. 2011). A pesar de que el proceso biológico que involucra la competencia entre árboles es más complejo de lo que se puede describir por una relación matemática (Daniels et al. 1986), índices de competencia son frecuentemente utilizados como una forma de representar la competencia natural, ayudando a expresar el estado competitivo de cada individuo en el bosque.

Así, con el desarrollo de diversos índices de competencia con los más variados niveles de complejidad, se destacan los que utilizan las variables diámetro a la altura del pecho (d) de los árboles objetivo y competidor (Lorimer 1983, Daniels et al. 1986, Corona y Ferrara 1989, Mugasha 1989), los que adicionan las distancias horizontales (Hegyi 1974, Rouvinen y Kuuluvainen 1997), los que agregan otras variables, como el área basal, altura y diámetro de copa (Ek y Monserud 1974, Glover y Hool 1979, Pukkala y Kolström 1987, Rouvinen y Kuuluvainen 1997). Hay también índices que se valen del área de proyección horizontal de copa sobre el suelo, de la superficie de intersección de copas del árbol objetivo y sus competidores (Gerrard 1969, Bella 1971, Álvarez Taboada et al. 2003, Castagneri et al. 2008) e índices que emplean el área de proyección de copa potencial sobre el suelo, determinada en árboles en crecimiento libre para la especie evaluada (Arney 1973).

En modelado del crecimiento de árbol individual, los índices de competencia constituyen la base de todo modelo, donde se utilizan para estimar el incremento y/o mortalidad durante una simulación (Téo et al. 2015), lo que ha permitido obtener ganancias en la eficiencia del modelo (Castro et al. 2014, Lustosa Junior et al. 2019). Sin embargo, dado que la habilidad de un índice puede variar según la especie en estudio, la región, las condiciones del bosque, etc., no hay consenso sobre una superioridad definida de un índice sobre otro, lo que requiere evaluaciones. Además, como la cuantificación de esta variable de competencia requiere la obtención de varias otras variables, a menudo difíciles de medir, la posibilidad de uso de ecuaciones que representen las distintas intensidades de competencia trae agilidad al proceso, facilitando las estimaciones.

En vista de esto, con la necesidad de conocer el comportamiento de distintos índices de competencia en relación con la especie de Araucaria angustifolia (Bertol.) Kuntze y permitir la agilidad de las aplicaciones, el presente estudio modela índices de competencia para árboles individuales en bosque natural. Específicamente se busca: (a) caracterizar los índices de competencia calculados; (b) ajustar modelos de regresión para describir la relación entre los índices de competencia en función del diámetro a la altura del pecho del árbol objetivo; (c) insertar y evaluar el comportamiento de la variable altura relativa en el modelo de competencia y verificar su influencia en cuanto al ajuste y a la precisión de las estimaciones.

\section{MÉTODOS}

Caracterización de las áreas de estudio. El trabajo se realizó en bosques naturales en los municipios de Lages (SC) y São Francisco de Paula (RS) (cuadro 1), siendo muestreados árboles creciendo bajo competencia. El clima de esos municipios, según la clasificación de Köppen, es subtropical húmedo, sin estación seca y con verano templado (Cfb) (Alvares et al. 2013).

En Lages, el estudio fue conducido en una propiedad rural particular con 83,5 hectáreas de bosque con $A$. angustifolia, ubicada cerca de la Hacienda Pedras Brancas, en el Planalto Central del estado de Santa Catarina. En el bosque muestreado fueron identificadas 28 especies conforme levantamiento realizado para el desarrollo del Plan de Manejo Forestal del sitio el año 2000.

En São Francisco de Paula, el estudio fue realizado en la Floresta Nacional (FLONA) de São Francisco de Paula, ubicada a $27 \mathrm{~km}$ distantes de la sede del municipio, en el nordeste del estado de Rio Grande do Sul, región de los

Cuadro 1. Ubicación y características climáticas de las áreas estudiadas.

Location and climatic characteristics of the studied areas.

\begin{tabular}{lccccc}
\hline \multicolumn{1}{c}{ Municipios } & Latitud S & Longitud W & Altitud (m) & TAM & PAM \\
\hline Lages & $-27^{\circ} 48^{\prime}$ & $-50^{\circ} 19^{\prime}$ & 987,0 & 15,2 & 1684,7 \\
São Francisco de Paula & $-29^{\circ} 26^{\prime}$ & $-50^{\circ} 35^{\prime}$ & 854,0 & 15,0 & 2016,4 \\
\hline
\end{tabular}

$\mathrm{TAM}=$ temperatura anual media $\left({ }^{\circ} \mathrm{C}\right) ; \mathrm{PAM}=$ precipitación anual media $(\mathrm{mm})$. 
Campos de Cima da Serra. En el Bosque con Araucaria de la FLONA fueron muestreados árboles pertenecientes a las parcelas $(1546,1537,1539,1543$ y 1545), instaladas por el Programa Brasileiro de Pesquisa Ecológicas de Longa Duração (PELD). Para las parcelas estudiadas, fueron identificadas 74 especies.

Los estimadores fitosociológicos de densidad, dominancia y frecuencia se calcularon permitiendo caracterizar las especies identificadas en ambos sitios y tener una idea de la biodiversidad de las comunidades (cuadro 2). El cálculo de estos parámetros horizontales se realizó en sus valores absolutos y relativos, y posteriormente se utilizó para calcular el valor de importancia (VI), de acuerdo con la literatura presentada por Müeller-Dombois y Ellenberg (1974). En ambos lugares, se destaca el alto VI observado para $A$. angustifolia, que representa la alta densidad, dominancia y frecuencia de la especie.

En un nuevo muestreo realizado por Schorr en 2018 para el mismo sitio de estudio de Lages-SC y utilizando los mismos procedimientos, se identificaron un total de 25 especies, y la especie de araucaria ha evolucionado a un VI del 53 \% (Schorr 2019). Esta disminución en el número de especies encontradas y el aumento en el dominio de A. angustifolia es probablemente el resultado de la competencia en el bosque, corroborando la necesidad de este tipo de estudio, así como la necesidad de pautas de gestión

Cuadro 2. Análisis fitosociológico horizontal: densidad, dominancia, frecuencia y valor de importancia de los sitios estudiados en el bosque con $A$. angustifolia.

Horizontal phytosociological analysis: density, dominance, frequency and importance value of the studied sites in the Araucaria Forest.

\begin{tabular}{|c|c|c|c|c|c|c|}
\hline $\mathrm{N}$ & Sitio & Nombre científico & DR & DoR & FR & VI \\
\hline 1 & \multirow{11}{*}{ Lages, SC. } & Araucaria angustifolia (Bertol.) Kuntze & 30,5 & 34,0 & 19,2 & 27,9 \\
\hline 2 & & Ocotea spp. & 27,2 & 29,6 & 13,7 & 23,5 \\
\hline 3 & & Sapium glandulosum (L.) Morong & 5,4 & 4,4 & 8,9 & 6,3 \\
\hline 4 & & Piptocarpha sp. & 4,5 & 3,5 & 8,9 & 5,6 \\
\hline 5 & & Lamanonia ternata Vell. & 5,7 & 5,6 & 2,7 & 4,7 \\
\hline 6 & & Lithraea brasiliensis Marchand & 2,9 & 2,7 & 6,8 & 4,2 \\
\hline 7 & & Styrax leprosus Hook. et Arn. & 3,7 & 3,5 & 2,7 & 3,3 \\
\hline 8 & & Myrsine umbellata Mart. & 2,9 & 2,2 & 3,4 & 2,9 \\
\hline 9 & & Ilex brevicuspis Reissek & 1,4 & 1,2 & 4,8 & 2,5 \\
\hline 10 & & Myrceugenia euosma (O.Berg) D. Legrand & 1,5 & 1,3 & 3,4 & 2,1 \\
\hline \multirow[t]{2}{*}{... 28} & & Otras spp. & 14,2 & 11,9 & 25,3 & 17,1 \\
\hline & \multirow{13}{*}{ São Francisco de Paula, RS. } & Total & 100,0 & 100,0 & 100,0 & 100,0 \\
\hline 1 & & Araucaria angustifolia (Bertol.) Kuntze & 26,2 & 67,6 & 15,4 & 36,4 \\
\hline 2 & & Blepharocalyx salicifolius (Kunth) O.Berg & 8,1 & 5,2 & 7,8 & 7,0 \\
\hline 3 & & Casearia decandra Jacq. & 7,7 & 1,7 & 7,5 & 5,6 \\
\hline 4 & & Sebastiania brasiliensis Spreng. & 7,3 & 1,4 & 7,0 & 5,2 \\
\hline 5 & & Ilex paraguariensis A. St.-Hil. & 4,1 & 1,8 & 5,1 & 3,6 \\
\hline 6 & & Matayba elaeagnoides Radlk. & 3,9 & 2,2 & 4,4 & 3,5 \\
\hline 7 & & Ilex brevicuspis Reissek & 2,7 & 2,2 & 3,2 & 2,7 \\
\hline 8 & & Cryptocarya aschersoniana $\mathrm{Mez}$ & 2,9 & 1,5 & 3,4 & 2,6 \\
\hline 9 & & Nectandra megapotamica (Spreng.) Mez & 2,6 & 1,5 & 3,2 & 2,4 \\
\hline 10 & & Luehea divaricata Mart. et Zucc. & 2,7 & 1,5 & 2,5 & 2,2 \\
\hline \multirow[t]{2}{*}{$\ldots 74$} & & Otras spp. & 31,8 & 13,4 & 40,5 & 28,6 \\
\hline & & Total & 100,0 & 100,0 & 100,0 & 100,0 \\
\hline
\end{tabular}

$\mathrm{N}$ = secuencia de especies; $\mathrm{DR}=$ densidad relativa (\%); DoR = dominancia relativa (\%); FR = frecuencia relativa (\%); VI = valor de importancia $(\%)$. 
y regulación de la estructura forestal que favorezcan la regeneración natural de las especies.

Levantamiento de los datos. Un total de 397 árboles de A. angustifolia, distribuidos en ocho clases de diámetro fueron seleccionados subjetivamente en el interior del bosque natural, buscando obtener árboles muestra en toda la amplitud diamétrica, considerando el menor centro de clase de $10 \mathrm{~cm}$ e intervalos entre clases diamétricas de $10 \mathrm{~cm}$, hasta la clase de $90 \mathrm{~cm}$. Los árboles de $A$. angustifolia seleccionados fueron denominados "árbol objetivo" y todos los árboles circunvecinos, independientemente de la especie, que ejerciesen competencia efectiva, también fueron demarcados y denominados "árboles competidores". De los 397 árboles objetivo, 308 (77,6 \%) fueron medidos en el municipio de Lages, SC, y los 89 árboles restantes (22,4\%) fueron medidos en la Flona de São Francisco de Paula, RS.

El criterio de selección de árboles competidores en relación con el árbol objetivo se basó en la dimensión de la copa y la altura de los árboles evaluados en cuanto a la capacidad de competir por luz y espacio de crecimiento. Los árboles circunvecinos, en un giro de $360^{\circ}$ del árbol objetivo, que tuviesen contacto lateral entre las copas, fueron considerados competidores. Además, los árboles por sobre el árbol objetivo, tanto si tocaban sus copas como si no, también se consideraban árboles competidores. De esa manera, el criterio de selección de árboles competidores puede ser representado por la expresión [1]:

$$
\operatorname{dist}_{\mathrm{ij}}<\left(\mathrm{rc}_{\mathrm{i}}+\mathrm{rc}_{\mathrm{j}}\right)
$$

En que: dist $_{\mathrm{ij}}=$ distancia horizontal entre el árbol objetivo (i) y el árbol competidor ( $\mathrm{j}$ ); $\mathrm{rc}_{\mathrm{i}}=$ radio de copa del árbol objetivo (i) en metros; $\mathrm{rc}_{\mathrm{j}}=$ radio de copa del árbol competidor (j) en metros.

A base de este criterio, se intentó seleccionar árboles competidores independientemente de su especie, pudiendo seleccionar, igualmente, árboles competidores de la misma especie (competencia intraespecífica) o especies distintas a araucaria (competencia interespecífica). Sin embargo, en ambos sitios estudiados, debido a la elevada dominancia de la especie de $A$. angustifolia, se encontró en los árboles objetivo la influencia predominante de la competencia intraespecífica.

En cada árbol objetivo y sus respectivos competidores, fueron medidos el diámetro a la altura del pecho (d), la altura total (h), la altura de inserción de copa (hic), los ocho radios de proyección de copa en la dirección de los puntos cardinales: norte $(\mathrm{N})$, noreste $(\mathrm{NE})$, este $(\mathrm{E})$, sureste (SE), sur $(\mathrm{S})$, suroeste $(\mathrm{SO})$, oeste $(\mathrm{O})$ y noroeste $(\mathrm{NO})$, y sus distancias horizontales (dist $t_{\mathrm{ij}}$ ).

La altura de inserción de copa fue definida como la altura desde el nivel del suelo hasta inserción de la copa viva. El diámetro fue medido con cinta diamétrica, en centímetros, y las distancias horizontales y alturas, ambas corregidas según la inclinación del terreno con el hipsómetro Vertex IV, en metros; así como el radio de copas de A. angustifolia, con precisión de centímetros. El diámetro de copa (dc) fue determinado doblándose el valor medio cuadrático de los ocho rayos medidos en la copa $(\mathrm{rcm})$. El área de proyección horizontal de las copas (APHC) de $A$. angustifolia fue calculada considerando la forma circular por $-\mathrm{APHC}=\pi \cdot \mathrm{rcm}^{2}$.

Índices de competencia. Los índices de competencia calculados (cuadro 3) fueron los que consideran entre el árbol objetivo (i) y sus respectivos competidores (j) las variables dimensionales: diámetro a la altura del pecho (d), distancias horizontales (dist), área basal del árbol individual (g), altura total (h), diámetro de copa $(\mathrm{dc})$, área de proyección horizontal de copa (APHC), área de proyección horizontal de copa potencial para A. angustifolia $\left(\mathrm{APHC}_{\mathrm{pot}}\right)$, área de superposición de copas (ASC) y radios de copa medios ( $\mathrm{rcm}$ ).

El cálculo de las áreas de superposición de copa (ASC) entre el árbol objetivo (i) y cada uno de los competidores (j) fue realizado por un algoritmo desarrollado en lenguaje Visual Basic, en la macro del editor de planilla Microsoft Excel 2013. Para eso, fue programada la expresión del área de superposición de copas presentada por varios autores (Gadow 2003, Álvarez Taboada et al. 2003), con el algoritmo adaptado para situaciones en que el APHC eran secantes entre sí o internas. Además, se revisaron los casos de copas tangentes externamente, internamente y concéntricas, las cuales no aparecieron en ninguno de los 1560 árboles analizados.

Máxima altura potencial (hmáx). Una variable importante que permite representar los poblamientos a lo largo de su vida es la altura dominante, que se define como la altura promedio de los mayores árboles de un poblamiento (Assmann 1970), y se caracteriza por tener una gran importancia biológica y ser poco influenciado por desbastes. Sin embargo, en el contexto de rodales disetáneos, esto no siempre es representativo, ya que, en este caso, generalmente se determina en los árboles más antiguos del poblamiento y generalmente se ubica en los mejores sitios (Finger 1992). De esa manera, en el presente estudio, se generó una variable análoga a la altura dominante, denominada "máxima altura potencial" (hmáx), utilizando técnicas de regresión cuántica (Davino et al. 2014), considerando la tendencia ajustada de la relación entre la altura/diámetro, equivalente a un cuantil de un $90 \%(\tau=0,9)$ de la variable dependiente (h). Aunque la relación altura/diámetro sufre el efecto de la edad y densidad, el uso de esta relación para clasificación de sitios en bosques naturales no puede ser rechazado (Temesgen et al. 2007). Este ajuste, con técnicas de regresión cuantílica, fue posible con auxilio de un algoritmo computacional desarrollado en el Sistema de Análisis Estadística (SAS) con la PROC NLP (SAS 2004). El comportamiento de la relación entre la hmáx con el d fue descripto con el modelo [2], adaptado de Michailoff (1943). 
Cuadro 3. Índices de competencia evaluados para Araucaria angustifolia en el bosque natural.

Competition indices evaluated for Araucaria angustifolia in the natural forest.

\begin{tabular}{|c|c|c|c|}
\hline Índices & Expresión & Variables & Fuente \\
\hline $\mathrm{IC} 1$ & $=D A P_{i}^{2} \cdot n /\left(\sum_{j=1}^{n} D A P_{j}^{2}\right)$ & d & Daniels et al. (1986) \\
\hline $\mathrm{IC} 2$ & $=\sum_{j=1}^{n}\left(D A P_{j}^{2} / D A P_{i}^{2}\right)$ & d & Corona y Ferrara (1989) \\
\hline IC3 & $=\left(\sum_{j=1}^{n} D A P_{j}\right) / D A P_{i}$ & d & Lorimer (1983) \\
\hline IC4 & $=\left(\sum_{j=1}^{n} D A P_{j} / D A P_{i}\right) / n$ & d & Mugasha (1989) \\
\hline IC5 & $=\sum_{j=1}^{n}\left[D A P_{j} /\left(D A P_{i}\right.\right.$. distij $\left.)\right]$ & d, dist & Hegyi (1974) \\
\hline IC6 & $=\sum_{j=1}^{n}\left[D A P_{j} /\left(D A P_{i} \cdot \operatorname{distij}^{0,5}\right)\right]$ & $\mathrm{d}$, dist & Hegyi (1974) - modificado \\
\hline IC7 & $=\sum_{j=1}^{n}\left[\left(D A P_{j} / D A P_{i}\right)^{2} /\right.$ distij $]$ & $\mathrm{d}$, dist & Rouvinen y Kuuluvainen (1997) \\
\hline IC8 & $=\sum_{j=1}^{n}\left(D A P_{j} /\right.$ distij $)$ & $\mathrm{d}$, dist & Rouvinen y Kuuluvainen (1997) \\
\hline IC9 & $=\sum_{j=1}^{n} g_{j}$ & G & Bal modificado \\
\hline $\mathrm{IC} 10$ & $=\sum_{j=1}^{n}\left(h_{i} / \bar{h}_{J}\right)$ & $\mathrm{H}$ & Glover y Hool (1979) \\
\hline $\mathrm{IC} 11$ & $=\sum_{j=1}^{n}\left(D A P_{i}^{2} h_{i} / \overline{D A P_{J}^{2} h_{J}}\right)$ & $\mathrm{d}, \mathrm{h}$ & Glover y Hool (1979) \\
\hline $\mathrm{IC} 12$ & $=\sum_{j=1}^{n}\left[\left(h_{j} / h_{i}\right) \operatorname{arctang}\left(h_{j} / \operatorname{distij}\right)\right]$ & $\mathrm{h}$, dist & Rouvinen y Kuuluvainen (1997) - modificado \\
\hline $\mathrm{IC} 13$ & $=\sum_{j=1}^{n} \operatorname{arctang}\left[\left(h_{j}-h_{i}\right) /\right.$ distij $]$ & $\mathrm{h}$, dist & Pukkala y Kolström (1987) \\
\hline $\mathrm{IC} 14$ & $=\sum_{j=1}^{n}\left[d c_{j} /\left(d c_{i}\right.\right.$. distij $\left.\left.^{0,5}\right)\right]$ & dc, dist & Hegyi (1974) - modificado \\
\hline IC15 & $=\sum_{j=1}^{n}\left[A P H C_{j} /\left(A P H C_{i} \cdot\right.\right.$ distij $\left.)\right]$ & APHC, dist & Álvarez Taboada et al. (2003) \\
\hline $\mathrm{IC} 16$ & $=\sum_{j=1}^{n}\left[A P H C_{j} / A P H C_{i}\right]$ & APHC & Castagneri et al. (2008) \\
\hline IC17 & $=\sum_{j=1}^{n}\left[A P H C_{j} h_{j} / A P H C_{i} h_{i}\right]$ & h, APHC & Castagneri et al. (2008) \\
\hline $\mathrm{IC} 18$ & $=\sum_{j=1}^{n}\left[A P H C_{j} h_{j} / A P H C_{i} h_{i}\right] / d i s t i j$ & $\mathrm{~h}, \mathrm{APHC}$, dist & Castagneri et al. (2008) \\
\hline IC19 & $=100 \sum_{j=1}^{n}\left[A S C_{i j}+A P H C_{p o t}\right] / A P H C_{p o t}$ & $\mathrm{APHC}_{\mathrm{pot}}, \mathrm{ASC}$ & Arney (1973) \\
\hline IC20 & $=\sum_{j=1}^{n}\left[A S C_{i j} / A P H C_{i}\right]$ & APHC, ASC & Gerrard (1969) \\
\hline $\mathrm{IC} 21$ & $=\sum_{j=1}^{n}\left[\left(A S C_{i j} D A P_{j}\right) /\left(A P H C_{i} D A P_{i}\right)\right]$ & d, APHC, ASC & Bella (1971) \\
\hline IC22 & $=\sum_{j=1}^{n}\left[A S C_{i j}\left(r c m_{j} h_{j} / r c m_{i} h_{i}\right)\right] / A P H C_{p o t}$ & $\mathrm{~h}, \mathrm{rcm}, \mathrm{APHC}_{\mathrm{pot}}, \mathrm{ASC}$ & Ek y Monserud (1974) \\
\hline
\end{tabular}

$\mathrm{d}=$ diámetro a la altura del pecho $(\mathrm{cm})$; dist $=$ distancia horizontal $(\mathrm{m}) ; \mathrm{g}=$ área basal del árbol individual $\left(\mathrm{m}^{2}\right) ; \mathrm{h}=$ altura total $(\mathrm{m}) ; \mathrm{dc}=$ diámetro de copa (m); APHC = área de proyección horizontal de la copa $\left(\mathrm{m}^{2}\right) ; \mathrm{APHC}_{\mathrm{pot}}=$ área de proyección horizontal de la copa potencial para A. angustifolia $\left(\mathrm{m}^{2}\right)$ - la ecuación: $\mathrm{dc}_{\mathrm{pot}}=4,8601+0,2038 . \mathrm{d}$, así: $\mathrm{APHC}_{\mathrm{pot}}=\pi\left(\mathrm{dc}_{\mathrm{pot}} / 2\right)^{2} ; \mathrm{ASC}=$ área de superposición de las copas $\left(\mathrm{m}^{2}\right) ;$ rcm = radio de copa medio $(\mathrm{m})$; i-ésimo árbol objetivo; $\mathrm{j}$-ésimo árbol competidor.

$$
h m a ́ x=1,3+\beta_{0} \exp \left(-\frac{\beta_{1}}{d}\right)+\varepsilon
$$

En que: hmáx = máxima altura potencial $(\mathrm{m}) ; \mathrm{d}=$ diámetro a la altura del pecho $(\mathrm{cm}) ; \beta 0, \beta 1=$ coeficientes de regresión estimados; $\varepsilon=$ error residual.

Del ajuste de la variable hmáx, se derivó la variable de altura relativa (hrel), como una razón entre la altura total (h) y la máxima altura potencial (hmáx) [3].

$$
\text { hrel }=\frac{\mathrm{h}}{\mathrm{hmáx}}
$$

Modelado de índices de competencia. Para describir la relación de los índices de competencia (IC) en función del d del árbol objetivo, se ajustó el modelo de potencia en la forma no linear [4]:

$$
I C=\beta_{0} d^{\beta_{1}}+\varepsilon
$$

Finalmente, se evaluó la contribución de la variable hrel en el modelo, además de la variable d, conforme la expresión [5].

$$
I C=\beta_{0} d^{\beta_{1}} \exp \left(\beta_{2} \text { hrel }\right)+\varepsilon
$$

En que: $\mathrm{IC}=$ índices de competencia; $\mathrm{d}=$ diámetro a la altura del pecho del árbol objetivo $(\mathrm{cm})$; hrel = altura relativa (h/hmáx); $\beta 0, \beta 1, \beta 2=$ coeficientes de regresión estimados; $\varepsilon=$ error residual. 
El modelo [4] inserta en la estimación de competencia la variación de la altura del árbol objetivo en relación con la máxima altura potencial para el mismo diámetro, vinculada, por lo tanto, a la idea de que cuanto mayor es la altura del árbol objetivo en relación con su potencial, menor es su grado de competencia.

Análisis de los datos. Las estadísticas fueron procesadas en el Sistema de Análisis Estadística (SAS 2004) y los modelos ajustados fueron evaluados mediante el coeficiente de determinación $\left(\mathrm{R}^{2}\right)$, al error patrón de la estimación en porcentaje (Syx \%) y a la probabilidad del valor de F del análisis de variancia.

\section{RESULTADOS}

Características de los índices de competencia. Cada uno de los índices de competencia calculados para los 397 árboles objetivo de A. angustifolia se caracterizan en el cuadro 4. Los valores del coeficiente de variación para los
22 índices de competencia fueron generalmente altos, lo que indica una alta variación en el estado competitivo de cada árbol individual. Además, la oscilación dentro de un rango de un $17,3 \%$ a un $650,2 \%$ entre índices proviene de la existencia de índices más y menos sensibles a las variaciones competitivas. Con respecto al índice 13 (IC13), los valores negativos verificados (cuadro 4) aparecieron cuando la altura del árbol objetivo fue menor que la de sus competidores.

Máxima altura potencial (hmáx). Con la determinación de la hmáx para los sitios estudiados, usando técnicas de regresión cuantílica (figura 1A), fue definida una nueva variable a ser usada en el modelado de los índices de competencia, denominada altura relativa (hrel), que deriva de la razón entre la altura del árbol objetivo y la hmáx estimada para el mismo d. En la figura 1B, se observa la distribución de frecuencias de las hrel de los árboles objetivo de araucaria con comportamiento similar de una distribución normal.

Cuadro 4. Resumen estadístico de los índices de competencia calculados para Araucaria angustifolia muestreadas en bosque natural. Statistical summary of the competition indices calculated for Araucaria angustifolia sampled in natural forests.

\begin{tabular}{crrrrrrr}
\hline Índices de competencia & Mínimo & Q1 & Media & Mediana & Q3 & Máximo & CV\% \\
\hline IC1 & 0,0032 & 0,0129 & 0,0219 & 0,0171 & 0,0254 & 0,1717 & 84,1 \\
IC2 & 0,2756 & 2,9939 & 7,6037 & 5,5728 & 9,9499 & 49,0338 & 90,2 \\
IC3 & 0,5250 & 2,9270 & 4,8242 & 4,4493 & 6,3861 & 14,9270 & 51,1 \\
IC4 & 0,4259 & 0,8893 & 1,3154 & 1,1675 & 1,6056 & 4,1888 & 45,5 \\
IC5 & 0,0802 & 0,4588 & 0,9084 & 0,7682 & 1,1875 & 6,6738 & 74,4 \\
IC6 & 0,2297 & 1,2094 & 2,0071 & 1,7875 & 2,5896 & 7,6860 & 55,5 \\
IC7 & 0,0527 & 0,4706 & 1,4511 & 0,9231 & 1,7761 & 16,2760 & 119,0 \\
IC8 & 1,8000 & 19,8475 & 29,9132 & 29,5466 & 39,0074 & 82,0129 & 45,7 \\
IC9 & 0,0076 & 0,3774 & 0,7182 & 0,6923 & 0,9996 & 2,5379 & 60,2 \\
IC10 & 0,3265 & 0,8661 & 0,9672 & 0,9605 & 1,0546 & 1,6606 & 17,3 \\
IC11 & 0,0343 & 0,3102 & 0,9514 & 0,6114 & 1,2117 & 8,2041 & 108,1 \\
IC12 & 0,6888 & 3,4579 & 4,9677 & 4,7188 & 6,4183 & 11,2471 & 43,6 \\
IC13 & $-4,8905$ & $-0,6872$ & 0,2270 & 0,2359 & 1,1765 & 4,4658 & 650,2 \\
IC14 & 0,1504 & 1,2267 & 1,9826 & 1,8226 & 2,5088 & 6,3517 & 53,1 \\
IC15 & 0,0227 & 0,4438 & 1,4023 & 0,9251 & 1,6887 & 12,0646 & 116,5 \\
IC16 & 0,1857 & 2,9447 & 7,5925 & 5,6378 & 8,5478 & 54,2230 & 98,3 \\
IC17 & 0,1881 & 2,9177 & 8,9063 & 6,0036 & 10,6166 & 90,0655 & 114,0 \\
IC18 & 0,0229 & 0,4564 & 1,6517 & 0,9486 & 1,9035 & 16,9717 & 129,8 \\
IC19 & 100,0 & 308,2 & 440,0 & 433,7 & 564,6 & 1205,1 & 45,7 \\
IC20 & 0,0 & 0,4357 & 0,9888 & 0,9433 & 1,4492 & 3,1824 & 65,5 \\
IC21 & 0,0 & 0,4743 & 1,4442 & 1,0333 & 1,9936 & 7,8866 & 94,4 \\
IC22 & 0,0 & 0,1761 & 0,7056 & 0,5130 & 1,0370 & 3,1804 & 93,8 \\
\hline
\end{tabular}

[IC1 a IC22] = índices de competencia; Q1 = primer cuartil; Q3 = tercer cuartil; CV\% = coeficiente de variación. 

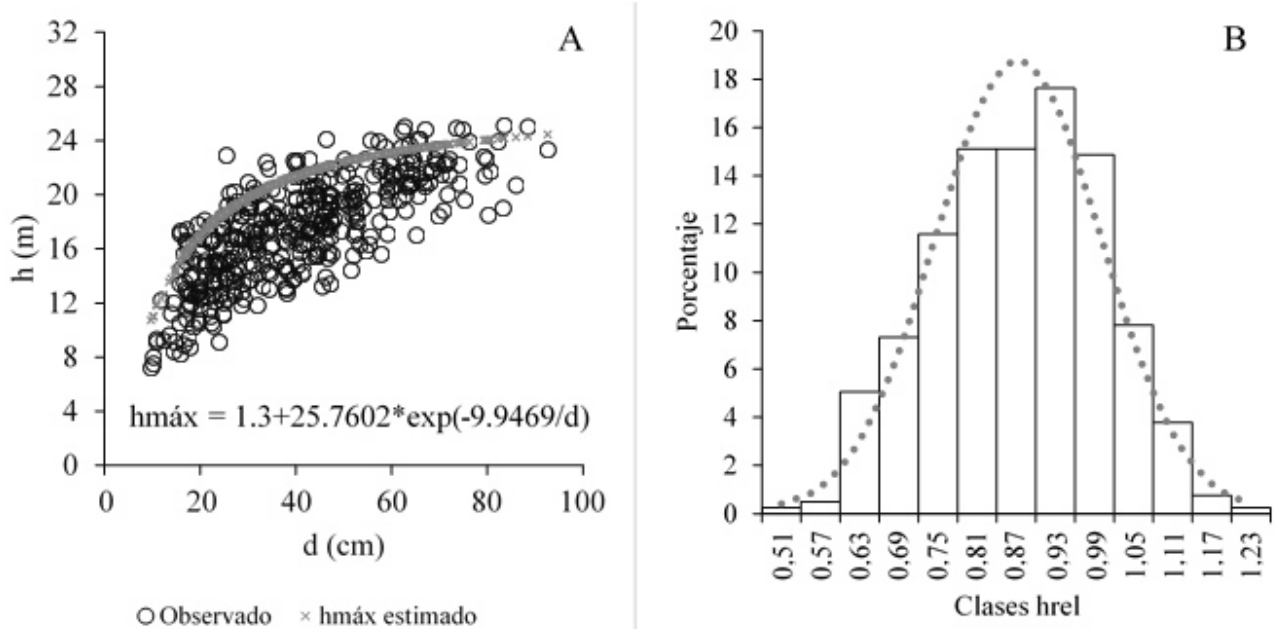

Figura 1. A) Estimación de la relación entre la máxima altura potencial (hmáx) en función del diámetro a la altura del pecho (d); B) Porcentaje de las frecuencias observadas en clases de altura relativa (hrel); para árboles de Araucaria angustifolia muestreados en bosque natural.

A) Estimation of the relationship between maximum potential height (hmáx) as a function of diameter at breast height (d). B) Percentage of observed frequencies in relative height (hrel) classes; for Araucaria angustifolia trees sampled in natural forests.

Modelado de índices de competencia. El ajuste del modelo [4] posibilitó describir la variación de los índices de competencia para árbol objetivo en función de su d. En tres de las ecuaciones probadas, no se encontró significancia para uno de los dos coeficientes de regresión estimados $(\alpha=5 \%)$, con todo, todas presentaron regresión con ajustes $\left(\mathrm{R}^{2}\right)$ variando de 0,010 a 0,546 y precisión (Syx \%) entre 15,0 \% y $600,2 \%$ (cuadro 5 ).

Al incluir la variable hrel además del d para describir la variación encontrada en cada índice de competencia (cuadro 6), se verificó que todas las ecuaciones presentaron regresión, pero no todos los coeficientes fueron significativos. En general, las ecuaciones tuvieron mejoría en cuanto a los criterios de ajuste y precisión, respecto de los determinados aplicando el modelo [4]. Por ejemplo, la ecuación de IC4 alcanzó ajuste $\left(\mathrm{R}^{2}\right)$ de 0,638 , seguida por la ecuación de IC7 con ajuste de 0,604 , y con el índice IC10 alcanzando la mayor precisión (Syx \%), alrededor del $14,6 \%$, pero con menor valor de ajuste.

La figura 2 presenta la dispersión de los valores observados y ajustados para los índices IC4 e IC7 en función de la variable d (2A) y de las variables d y hrel (2B), ilustrando que a medida que los árboles aumentan de diámetro, hay una disminución en la intensidad de la competencia representada por los índices.

\section{DISCUSIÓN}

Características de los índices de competencia. Los índices de competencia expresan la influencia de los árboles vecinos en el espacio vital de cada árbol objetivo, variando a medida que este entorno se ve comprometido por el crecimiento (tamaño) y la ocupación del espacio. En cuanto a la interpretación de los valores de los índices, inicialmente es necesario considerar la metodología de cálculo de cada índice, ya que pueden representar la competencia directa o inversamente. La representación es directa cuando el tamaño de los árboles competidores se expresa en el numerador y el tamaño del árbol objetivo en el denominador (por ejemplo, índices IC2, IC5, IC9 e IC15), y cuanto menor es el valor de estos índices, menor es la competencia sobre el árbol objetivo. Otros índices tienen la característica de representar la competencia inversamente, cuando es el tamaño del árbol objetivo que aparece en el numerador (índices IC1, IC10 e IC11) y, por lo tanto, cuanto menor sea el valor de estos índices, mayor será la competencia representada.

Sin embargo, independientemente de la metodología para calcular los índices, se interpreta que cuanto mayor sea el tamaño de los competidores en relación con el árbol objetivo, así como su proximidad (cuando se trata de los índices dependientes de la distancia), mayor será la competencia sobre el árbol objetivo.

Con respecto a las características de los índices IC19, IC20, IC21 e IC22 (cuadro 4), se identificó que el valor mínimo de 100,0 para el IC19 y cero para los IC20, IC21 e IC22 resultaron de nueve árboles objetivo de $A$. angustifolia que tenían sus copas asimétricas, lo que provocó la reducción del radio medio de copa usado en el cálculo, reduciendo este valor hasta el punto de que no había más intersección de copas, contradiciendo los resultados encontrados en campo. Aunque se midieron ocho radios de copa, el método no permitió describir totalmente esa dimensión para la especie evaluada. En este estudio, ese resultado fue considerado como natural y de posible ocurrencia en campo, y otros métodos de cálculo de áreas de proyección hori- 
zontal de copa no han sido explorados, como los polígonos de Voronoi (Gspaltl et al. 2012), entre otros, lo que tal vez pudiese proporcionar resultados más eficaces.

Modelado de índices de competencia. Los resultados del presente trabajo permitieron describir los índices de competencia del árbol objetivo para distintos niveles de complejidad de cálculo (cuadro 3), con las ecuaciones ajustadas, apenas midiendo el d (cuadro 5) y con la altura medida del árbol objetivo (cuadro 6) para obtener mayor precisión en las predicciones.

Fue posible verificar que, de modo general, índices de competencia independientes y dependientes de la distancia que consideran en el cálculo apenas las variables d del árbol objetivo, d de sus competidores, distancias horizontales y eventualmente la variable altura, presentaron desempeño superior a los índices que evalúan va- riables de dimensión de copa y de superposición de áreas de copa entre el árbol objetivo y competidores, difiriendo de lo que fue sugerido por autores como Canham et al. (2004) y Coates et al. (2009). De esta forma, para el modelado del crecimiento de la especie $A$. angustifolia, los primeros índices serían los más indicados debido a la simplicidad y a la practicidad de aplicación en campo en bosque natural.

Además, la competencia sufrida por los árboles objetivo, estimada según el IC4 (figura 2A) e IC7 (figura 2C) como una función del d, probó que, con el aumento del d del árbol objetivo, sucede la disminución de la intensidad de la competencia. Los árboles más grandes, en general, por alcanzar estratos superiores de los bosques, tienen menor competencia (Cunha 2009). En consecuencia, árboles pequeños, ubicados en el sotobosque, estarán bajo mayores influencias competitivas.

Cuadro 5. Coeficientes estimados y estadísticas de ajuste y precisión de los índices de competencia calculados en función del diámetro a la altura del pecho (d) del árbol objetivo para Araucaria angustifolia muestreadas en bosque natural.

Estimated coefficients and statistics of adjustment and accuracy of the competition indices calculated according to the diameter at breast height (d) of the central tree for Araucaria angustifolia sampled in natural forests.

\begin{tabular}{cccccc}
\hline Índices de competencia & $\beta_{0}$ & $\beta_{1}$ & $\mathrm{R}^{2}$ & Syx\% & Prob.>F \\
\hline IC1 & 0,0111 & 0,1880 & 0,011 & 83,6 & $<0,0001$ \\
IC2 & 183,0338 & $-0,9066$ & 0,315 & 74,7 & $<0,0001$ \\
IC3 & 15,5515 & $-0,3288$ & 0,106 & 48,4 & $<0,0001$ \\
IC4 & 13,5422 & $-0,6622$ & 0,546 & 30,7 & $<0,0001$ \\
IC5 & 18,0592 & $-0,8540$ & 0,358 & 59,7 & $<0,0001$ \\
IC6 & 14,4389 & $-0,5581$ & 0,267 & 47,6 & $<0,0001$ \\
IC7 & 137,5439 & $-1,3128$ & 0,419 & 90,9 & $<0,0001$ \\
IC8 & 26,5090 & $0,0335 \mathrm{~ns}$ & 0,001 & 45,8 & $<0,0001$ \\
IC9 & 0,0661 & 0,6526 & 0,253 & 52,1 & $<0,0001$ \\
IC10 & 0,4966 & 0,1845 & 0,247 & 15,0 & $<0,0001$ \\
IC11 & 0,0069 & 1,3216 & 0,271 & 92,3 & $<0,0001$ \\
IC12 & 4,3687 & $0,0356 \mathrm{~ns}$ & 0,002 & 43,6 & $<0,0001$ \\
IC13 & $279,5 \mathrm{~ns}$ & $-1,9371$ & 0,151 & 600,2 & $<0,0001$ \\
IC14 & 9,3463 & $-0,4369$ & 0,178 & 48,2 & $<0,0001$ \\
IC15 & 56,3952 & $-1,0561$ & 0,274 & 99,4 & $<0,0001$ \\
IC16 & 95,3375 & $-0,7169$ & 0,163 & 90,0 & $<0,0001$ \\
IC17 & 212,1718 & $-0,9030$ & 0,199 & 102,2 & $<0,0001$ \\
IC18 & 127,1095 & $-1,2490$ & 0,321 & 107,1 & $<0,0001$ \\
IC19 & 171,1700 & 0,2610 & 0,076 & 44,0 & $<0,0001$ \\
IC20 & 2,4286 & $-0,2518$ & 0,040 & 64,3 & $<0,0001$ \\
IC21 & 20,2096 & $-0,7475$ & 0,200 & 84,5 & $<0,0001$ \\
IC22 & 3,3465 & $-0,4373$ & 0,068 & 90,7 & $<0,0001$ \\
\hline
\end{tabular}

[IC1 a IC22] = índices de competencia; $\beta_{0} \beta_{1}=$ coeficientes de regresión estimados; $\mathrm{R}^{2}=$ coeficiente de determinación; Syx $\%=$ error patrón de la estimación en porcentaje; Prob. $>\mathrm{F}=$ probabilidad de significancia para el valor de F; ns = coeficiente de regresión estimado no significante a $\alpha=5 \%$. 
La misma relación se logró para los mismos índices con la inclusión de la variable hrel en el modelo (figura 2B y 2D), lo que ocasionó mejor ajuste y precisión en las estimaciones. La inclusión de la variable hrel además del d para describir la variación encontrada en cada índice de competencia se vuelve bastante importante en el contexto de que, en general, cuanto más se suprima el árbol, mayor será la competencia ejercida sobre él, y viceversa. En este caso, cuanto mayor sea la altura del árbol objetivo en relación con su altura potencial máxima, menor será el grado de competencia representado.

La generación de estas ecuaciones es importante, principalmente cuando se pretende determinar el grado de competencia medio para el árbol objetivo. Esta manera trae agilidad al proceso, dispensando la necesidad de determinación de variables de difícil obtención, tanto para árboles objetivos como para los competidores. Esas varia- bles pueden contener errores elevados, dependiendo del equipamiento usado, mayor tiempo para medición y costes adicionales, principalmente cuando levantadas en bosques mixtos y disetáneos. Variables que cuantifican la dimensión de copa, áreas de superposición de copas, entre otras, son ejemplos de variables que no se miden habitualmente para todos los árboles en inventario de bosque natural. Se confirma, por lo tanto, la importancia en alcanzar valores de índices de competencia por medio de ecuaciones.

\section{CONCLUSIONES}

Entre los índices de competencia independientes y dependientes de la distancia probados, aquellos que consideran en el cálculo solo las variables diámetro del árbol objetivo, diámetro de sus competidores, distancias horizontales, y eventualmente la variable altura, son los más

Cuadro 6. Coeficientes estimados y estadísticas de ajuste y precisión de los índices de competencia calculados en función del diámetro a la altura del pecho (d) y altura relativa (hrel) del árbol objetivo para Araucaria angustifolia muestreadas en bosque natural.

Estimated coefficients and statistics of adjustment and precision of the competition indices calculated according to the diameter at breast height (d) and relative height (hrel) of the central tree for Araucaria angustifolia sampled in natural forests.

\begin{tabular}{ccccccc}
\hline Índices de competencia & $\beta_{0}$ & $\beta_{1}$ & $\beta_{2}$ & $\mathrm{R}^{2}$ & Syx\% & Prob.>F \\
\hline IC1 & 0,0432 & 0,3248 & $-2,2354$ & 0,110 & 79,5 & $<0,0001$ \\
IC2 & 53,0641 & $-1,0737$ & 2,1171 & 0,480 & 65,2 & $<0,0001$ \\
IC3 & 7,5770 & $-0,4147$ & 1,2047 & 0,213 & 45,5 & $<0,0001$ \\
IC4 & 7,5674 & $-0,7200$ & 0,9438 & 0,638 & 27,4 & $<0,0001$ \\
IC5 & 7,2029 & $-0,9750$ & 1,5680 & 0,468 & 54,4 & $<0,0001$ \\
IC6 & 6,4945 & $-0,6503$ & 1,3208 & 0,386 & 43,6 & $<0,0001$ \\
IC7 & 34,8671 & $-1,5832$ & 2,6213 & 0,605 & 75,0 & $<0,0001$ \\
IC8 & 14,8023 & $-0,05062 \mathrm{~ns}$ & 1,0412 & 0,082 & 43,9 & $<0,0001$ \\
IC9 & 0,0375 & 0,0539 & 1,1539 & 0,305 & 50,3 & $<0,0001$ \\
IC10 & 0,4185 & 0,1638 & 0,2909 & 0,287 & 14,6 & $<0,0001$ \\
IC11 & 0,0125 & 1,4170 & $-1,1127$ & 0,286 & 91,5 & $<0,0001$ \\
IC12 & 3,9873 & $0,0232 \mathrm{~ns}$ & $0,1611 \mathrm{~ns}$ & 0,004 & 43,6 & $<0,0001$ \\
IC13 & $314,2 \mathrm{~ns}$ & $-1,8930$ & $-0,3048 \mathrm{~ns}$ & 0,151 & 600,8 & $<0,0001$ \\
IC14 & 4,3604 & $-0,5257$ & 1,2661 & 0,293 & 44,8 & $<0,0001$ \\
IC15 & 14,3279 & $-1,2532$ & 2,3769 & 0,417 & 89,2 & $<0,0001$ \\
IC16 & 29,7188 & $-0,8499$ & 1,9118 & 0,267 & 84,3 & $<0,0001$ \\
IC17 & 79,0682 & $-1,0348$ & 1,6926 & 0,267 & 97,8 & $<0,0001$ \\
IC18 & 39,9865 & $-1,4532$ & 2,1438 & 0,426 & 98,6 & $<0,0001$ \\
IC19 & 153,9635 & 0,2431 & $0,2019 \mathrm{~ns}$ & 0,0790 & 43,9 & $<0,0001$ \\
IC20 & 2,0025 & $-0,2814$ & $0,3531 \mathrm{~ns}$ & 0,0446 & 64,2 & $<0,0001$ \\
IC21 & 9,2319 & $-0,8545$ & 1,3571 & 0,2507 & 81,9 & $<0,0001$ \\
IC22 & 4,0193 & $-0,4093$ & $-0,3364$ & 0,0705 & 90,7 & $<0,0001$ \\
\hline
\end{tabular}

[IC1 a IC22] = índices de competencia; $\beta_{0} \beta_{2}=$ coeficientes de regresión estimados; $\mathrm{R}^{2}=$ coeficiente de determinación; Syx $\%=$ error patrón de la estimación en porcentaje; Prob. $>\mathrm{F}=$ probabilidad de significancia para el valor de F; ns = coeficiente de regresión estimado no significante a $\alpha=5 \%$. 

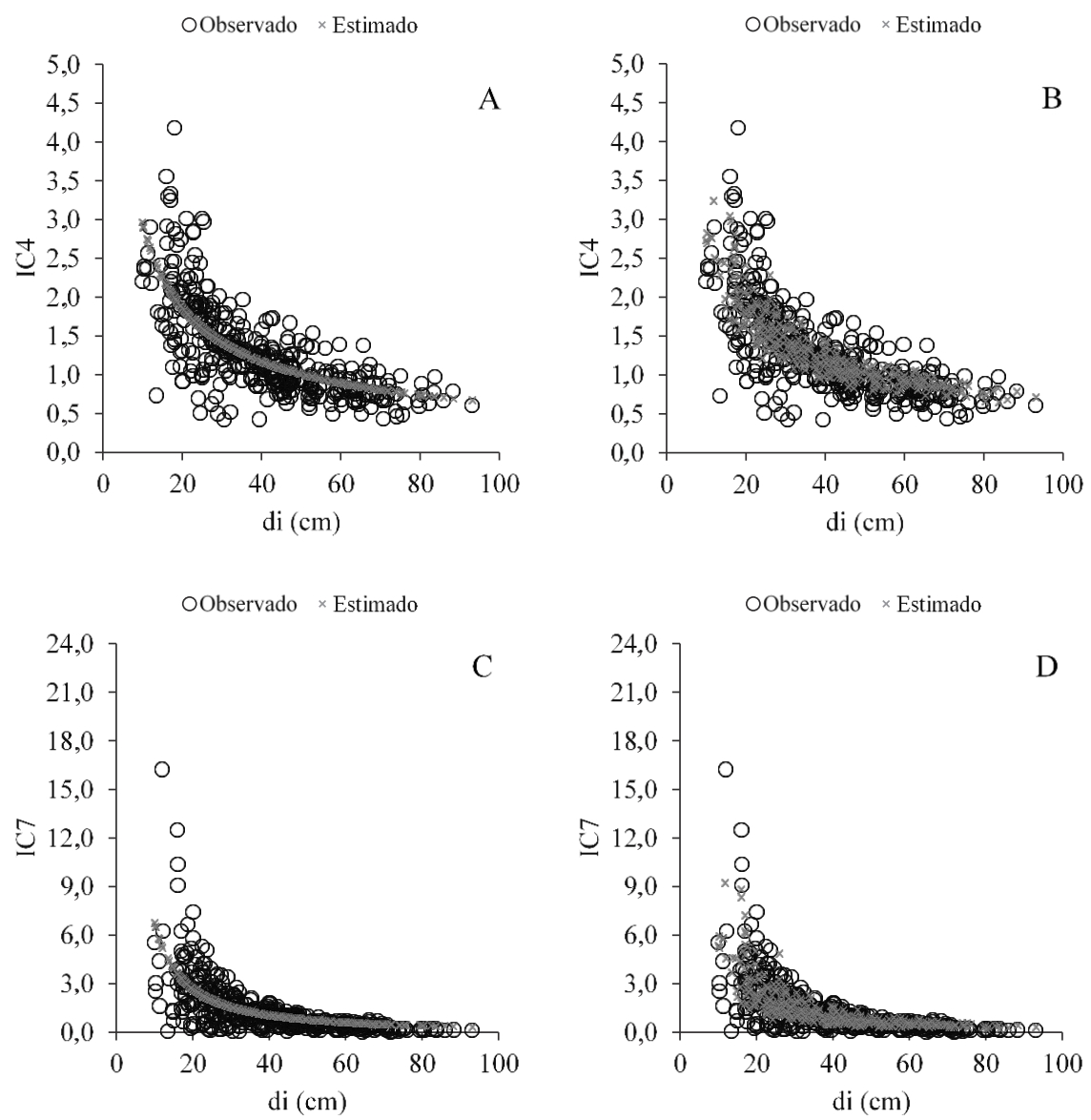

Figura 2. A) y C) Valores observados y estimados de los índices de competencia (IC4) e (IC7) en función del diámetro a la altura del pecho (d) del árbol objetivo. B) y (D) Valores observados y estimados de los índices de competencia (IC4) e (IC7) en función del diámetro a la altura del pecho (d) y altura relativa (hrel) del árbol objetivo; para árboles de Araucaria angustifolia muestreados en bosque natural.

A) and C) Observed and estimated values of competition indices (IC4) and (IC7) according to the diameter at breast height (d) of the central tree. B) and D) Observed and estimated values of competence indices (IC4) and (IC7) according to the diameter at breast height (d) and relative height (hrel) of the central tree; for Araucaria angustifolia trees sampled in natural forests.

indicados para el modelado del crecimiento de $A$. angustifolia, debido a la simplicidad y a la practicidad de aplicación en bosque natural.

Las ecuaciones desarrolladas, destacando aquellas correspondientes a los índices IC4 e IC7, se presentaron como buenas representaciones del grado de competencia a nivel del árbol individual de A. angustifolia, basadas solo en la variable diámetro, y con mejoras adicionales con la inclusión de la variable altura relativa, para los dos sitios estudiados en el sur de Brasil.

Además, otros sitios en la región sur de Brasil, así como a lo largo de toda la distribución de la especie, aún necesitan ser investigados para confirmar la aplicabilidad de estas ecuaciones en toda su amplitud, y se sugieren para futuras investigaciones.

Este trabajo contribuye con aportes para la inclusión de índices de competencia en el contexto del modelado a nivel de árbol individual en bosques mixtos disetáneos.

\section{REFERENCIAS}

Alvares CA, JL Stape, PC Sentelhas, JLM Gonçalves, G Sparovek. 2013. Köppen's climate classification map for Brazil. Meteorologische Zeitschrift 22: 711-728. DOI: 10.1127/0941-2948/2013/0507

Álvarez Taboada MFA, MB Anta, JG Varela, JGA Álvarez González. 2003. Influencia de la competencia en el crecimiento en sección en Pinus radiata D. Don. Investigación Agraria: Sistemas Recursos Forestales 12: 25-35.

Arney JD. 1973. Tables for quantifying competitive stress on individual trees. Victoria, Canada. Pacific Forest Research Centre, Canadian Forestry Service. 45 p.

Assmann E. 1970. The principles of forest yield study. Oxford, UK. Pergamon Press. 506 p.

Bella IE. 1971. A new competition model for individual trees. Forest Science 17: 364-372. DOI: https://doi.org/10.1093/ forestscience/17.3.364

Burkhart HE, M Tomé. 2012. Modeling Forest Trees and Stands. Dordrecht, The Netherlands. Springer. 457 p. 
Canham CD, PT Lepage, KDA Coates. 2004. A neighborhood analysis of canopy tree competition: effects of shading versus crowding. Canadian Journal of Forest Research 34: 778-787. DOI: https://doi.org/10.1139/x03-232

Castagneri D, G Vacchiano, E Lingua, R Motta. 2008. Analysis of intraspecific competition in two subalpine Norway spruce (Picea abies (L.) Karst.) stands in Paneveggio (Trento, Italy). Forest Ecology and Management 255: 651-659.

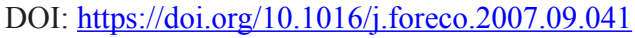

Castro RVO, CPB Soares, HG Leite, AL Souza, FB Martins, GS Nogueira, M Oliveira, F Silva. 2014. Competição em nível de árvore individual de uma floresta estacional semidecidual. Silva Lusitana 22(1): 43-66.

Coates KD, CD Canham, CT Lepage. 2009. Above versus below ground competitive effects and responses of a guild of temperate tree species. Journal of Ecology 97: 118-130. DOI: https://doi.org/10.1111/j.1365-2745.2008.01458.x

Corona P, A Ferrara. 1989. Individual competition indices for conifer plantations. Agriculture, Ecosystems and Environment 27: 429-437. DOI: https://doi.org/10.1016/B978-0444-88610-1.50039-5

Craine JM, R Dybzinski. 2013. Mechanisms of plant competition for nutrients, water and light. Functional Ecology 27: 833840. DOI: https://doi.org/10.1111/1365-2435.12081

Cunha TA. 2009. Modelagem do incremento de arvores individuais de Cedrela odorata L. na Floresta Amazônica. Dissertação Mestrado. Santa Maria, Brasil. Universidade Federal de Santa Maria. 88 p.

Daniels RF, HE Burkhart, TR Clason. 1986. Comparison of competition measures for predicting growth of loblolly pine trees. Canadian Journal for Forest Research 16: 12301237. https://doi.org/10.1139/x86-218

Davino C, M Furno, D Vistocco. 2014. Quantile Regression: Theory and Applications. Chichester, UK. Wiley. 260 p.

Ek AR, RA Monserud. 1974. FOREST: a computer model for simulating the growth and reproduction of mixed species forest stands. Madison, USA. University of Wisconsin, College of Agriculture and Life Science. 90 p.

Finger CAG. 1992. Fundamentos de Biometria Florestal. Santa Maria, Brasil. UFSM/CEPEF/FATEC. 269 p.

Gadow KV. 2003. Waldstruktur und Wachstum. Beilage zur Vorlesung im Wintersemester 2003/2004. Vorlesungsmanuskript für Studenten. Göttingen, Germany. Universitätsverlag Göttingen. 241 p.

Gerrard DI. 1969. Competition quotient: a new measure for the competition affecting individual forest trees. Agricultural Experiment Station, Michigan State University. 32 p.

Glover GR, JN Hool. 1979. A basal area ratio on predictor of loblolly pine plantations mortality. Forest Science 25(2): 275282. DOI: https://doi.org/10.1093/forestscience/25.2.275

Gspaltl M, H Sterba, KL O'hara. 2012. The relationship between available area efficiency and area exploitation index in an evenaged coast redwood (Sequoia sempervirens) stand. Forestry 85: 567-577. DOI: https://doi.org/10.1093/forestry/cps052

Hegyi FA. 1974. Simulation model for managing jack-pine stands. In Royal College of Forestry ed. Growth models for tree and stand population. Stockholm, Sweden. p. 74-90.

Kuehne C, AR Weiskittel, J Waskiewicz. 2019. Comparing performance of contrasting distance-independent and distance dependent competition metrics in predicting individual tree diameter increment and survival within structurally-heterogeneous, mixed-species forests of Northeastern United States. Forest Ecology and Management 433: 205-216. DOI: https://doi.org/10.1016/j.foreco.2018.11.002

Lorimer CG. 1983. Tests of age independent competition indices for individual trees in natural hardwood stands. Forest Ecology and Management 6: 343-360. DOI: https://doi. org/10.1016/0378-1127(83)90042-7

Lustosa Junior IM, RVO Castro, RO Gaspar, JBC Araújo, FG Aquino. 2019. Competition Indexes to Evaluate Tree Growth in a Semi-Deciduous Seasonal Forest. Floresta e Ambiente 26(4): e20160107. http://dx.doi. org/10.1590/2179-8087.010716

Michailoff I. 1943. Zahlenmäßiges Verfahren für die Ausführung der Bestandeshöhenkurven. Forstwissenschaftliches Centralblatt und Tharandter Forstliches Jahrbuch 6:273-279.

Mueller-Dombois D, H Ellenberg. 1974. Aims and methods of vegetation ecology. New York, USA. John Wiley. 547 p.

Mugasha AG. 1989. Evaluation of Simple Competition Indices for the Prediction of Volume Increment of Young Jack Pine and Trembling Aspen Trees. Forest Ecology and Management 26: 227-235. https://doi.org/10.1016/0378$\underline{1127(89) 90123-0}$

Pukkala T, T Kolström. 1987. Competition indices and the prediction of radial growth in Scots pine. Silva Fennica 21: 55-67. DOI: https://doi.org/10.14214/sf.a15463

Rouvinen S, T Kuuluvainen. 1997. Structure and asymmetry of tree crowns in relation to local competition in a natural mature Scots pine forest. Canadian Journal for Forest Research 27: 890-902. DOI: https://doi.org/10.1139/x97-012

SAS. 2004. The SAS System for Windows. Cary, USA. SAS Institute.

Shorr LPB. 2019. Dinâmica e relações alométricas para espécies arbóreas em Floresta Ombrófila Mista sob regime de nãomanejo no sul do Brasil. Dissertação Mestrado. Lages, Brasil. Universidade do Estado de Santa Catarina. 71 p.

Temesgen H, D Hann, V Monleon. 2007. Regional Height Diameter Equations for Maior Tree Species of Southwest Oregon. Western Journal of Applied Forestry 22: 213-219. DOI: https://doi.org/10.1093/wjaf/22.3.213

Téo SJ, A Figueiredo Filho, C Lingnau. 2015. Análise espacial do estresse competitivo, incremento diamétrico e estrutura de uma Floresta Ombrófila Mista, Irati, PR. Floresta 45(4): 681-694. DOI: 10.5380/rf.v45i4.39939

Weiskittel AR, DW Hann, JA Kershaw Jr, JK Vanclay. 2011. Forest Growth and Yield Modeling. 2nd ed. Chicester, UK. Wiley-Blackwell. 415 p.

Zhang Y, X Deng, Y Huang, Y Li, W Xiang, W Yan. 2019. Quantification of individual tree competition index taking Chinese-fir plantations in subtropical low hilly area as an example. Polish Journal of Ecology 67: 1-16. DOI: https:// doi.org/10.3161/15052249PJE2019.67.1.001 
